\title{
Almost $P_{p}$-continuous functions
}

\author{
Shadya M. Mershkhan \\ Department of Mathematics, Faculty of Science, University of Zakho, Iraq \\ Received: 25 September 2018, Accepted: 9 July 2019 \\ Published online: 25 December 2019.
}

\begin{abstract}
This paper is aimed to introduce a new class of functions called almost $P_{p}$-continuous functions by using $P_{p}$-open sets in topological spaces. Also some properties and characterizations are studied.
\end{abstract}

Keywords: $P_{p}$-open, preopen, almost $P_{p}$-continuous, almost precontinuous.

\section{Introduction and Preliminaries}

In 1982, Mashhour et al [13] defined a new class of sets called preopen sets and almost precontinuous functions is defined in [16]. In [10] the concept of $P_{p}$-open sets is introduced. In the present paper, we introduce and investigate the concept of almost $P_{p}$-continuous functions. It will be shown that almost $P_{p}$-continuity is weaker than $P_{p}$-continuity mentioned in [19], but it is stronger than almost precontinuity.

Throughout the present paper, a space $X$ always means a topological space on which no separation axiom is assumed unless explicitly stated. Let $A$ be a subset of a space $X$. The closure and interior of A with respect to $X$ are denoted by $C l(A)$ and $\operatorname{Int}(A)$ respectively. A subset $A$ of a space $X$ is said to be preopen [13] (resp., semi-open [11], $\alpha$-open [17], $\beta$-open [1] and regular open [22]), if $A \subseteq \operatorname{Int}(C l(A))$ (resp., $A \subseteq C l(\operatorname{Int}(A)), A \subseteq \operatorname{Int}(C l(\operatorname{Int}(A)), A \subseteq C l(\operatorname{Int}(C l(A))$ and $A=\operatorname{Int}(C l(A)))$. The complement of a preopen (resp., semi-open, $\alpha$-open, $\beta$-open and regular open ) set is said to be preclosed (resp., semi-closed, $\alpha$-closed, $\beta$-closed and regular closed). The family of all preopen (resp., semi-open, $\alpha$-open, $\beta$-open and regular open) subsets of $X$ is denoted by $P O(X)$ (resp., $S O(X), \alpha O(X), \beta O(X)$ and $R O(X)$ ). A subset $A$ of a space $X$ is called $\delta$-open (resp., $\theta$-open) if for each $x \in A$, there exists an open set $G$ such that $x \in G \subseteq \operatorname{Int}(C l(G)) \subseteq A$ (resp., $x \in G \subseteq C l(G) \subseteq A$ ). In 1968,Velicko [23] defined the concepts of $\delta$-open and $\theta$-open sets in $X$ (denoted by $\delta O(X)$ and $\theta O(X)$ respectively).

A function $f: X \rightarrow Y$ is said to be precontinuous [13] (resp., super continuous [15], strongly $\theta$-continuous [12]) if $f^{-1}(V)$ is preopen (resp., $\delta$-open, $\theta$-open) in $X$ for every open set $V$ of $Y$. A function $f: X \rightarrow Y$ is said to be almost precontinuous [16] if the inverse image of each regular open subset of $Y$ is preopen in $X$. A function $f: X \rightarrow Y$ is said to be $\theta$-continuous [7] (resp., almost strongly $\theta$-continuous [18]) if for each $x \in X$ and each open set $V$ of $Y$ containing $f(x)$, there exists an open set $U$ of $X$ containing $x$ such that $f(C l U) \subseteq C l V$ (resp., $f(C l U) \subseteq s C l V$ ).

Definition 1.[10] A subset $A$ of a space $X$ is called $P_{p}$-open, if for each $x \in A \in P O(X)$, there exists a preclosed set $F$ such that $x \in F \subseteq$ A.The complement of a $P_{p}$-open is $P_{p}$-closed. The family of all $P_{p}$-open subsets of a topological space $(X, \tau)$ is denoted by $P_{p} O(X, \tau)$ or $P_{p} O(X)$. 
The intersection of all $P_{p}$-closed (resp., preclosed, semi-closed, $\alpha$-closed and $\delta$-closed) sets of $X$ containing $A$ is called the $P_{P}$-closure (resp. preclosure, semi-closure, $\alpha$-closure and $\delta$-closure) of $A$ and is denoted by $P_{p} C l(A)($ resp. $p C l(A)$ , $s C l(A), \alpha C l(A)$ and $C l \delta(A)$ ). The union of all $P_{P}$-open (resp., preopen, semi-open, $\alpha$-open and $\delta$-open) sets of $X$ contained in $A$ is called the $P_{p}$-interior (resp., preinterior, semi-interior, $\alpha$-interior and $\delta$-interior) of $A$ and is denoted by $P_{p} \operatorname{Int}(A)($ resp. $p \operatorname{Int}(A), \operatorname{sint}(A), \alpha \operatorname{Int}(A)$ and $\delta \operatorname{Int}(A))$.

Definition 2. A space $X$ is said to be:

(1) locally indiscrete [5] if every open subset of $X$ is closed.

(2) Pre- $R_{0}[4]$, if $U$ is a preopen set and $x \in U$, then $\operatorname{PCl}(\{x\}) \subseteq U$.

(3) Pre- $T_{1}[9]$ if for each pair of distinct points $x, y$ of $X$, there exist two preopen sets one containing $x$ but not $y$ and other containing y but not $x$.

Definition 3. [21] A space $X$ is said to be pre-regular if for each preclosed set $F$ and each point $x \notin F$, there exist disjoint preopen sets $U$ and $V$ such that $x \in U$ and $F \subseteq V$

Proposition 1. [10] The following statements are true:

(1) If a space $X$ is pre- $T_{1}$, then $P O(X)=P_{p} O(X)$.

(2) If a space $X$ is pre-regular, then $\tau \subseteq P_{p} O(X)$.

(3) If a space $(X, \tau)$ is locally indiscrete, then $P O(X)=P_{p} O(X)$.

Corollary 1. [14] For any space $X$, if $X$ is pre- $R_{0}$, then $P O(X)=P_{p} O(X)$.

Lemma 1. Let $X$ be a space. The following statements are true:

(1) $R \in R O(X)$ and $P \in P O(X)$, then $R \cap P \in P O(P)$ [5].

(2) Let $A \subseteq X$. Then $A \in P O(X, \tau)$ if and only if $\operatorname{sCl}(A)=\operatorname{IntCl}(A)[8]$.

(3) $A$ is $\beta$-open if and only if $\mathrm{Cl}(A)$ is regular closed [3].

Lemma 2. Let $A$ be a subset of $X$. Then:

(1) If $A \in S O(X)$, then $p C l(A)=C l(A)[6]$.

(2) If $A \in \beta O(X)$, then $\alpha C l(A)=C l(A)[2]$.

(3) If $A \in \beta O(X)$, then $C l_{\delta}(A)=C l(A)[24]$.

Definition 4. [10] A function $f: X \rightarrow Y$ is called $P_{p}$-continuous at a point $x \in X$ if for each open set $V$ of $Y$ containing $f(x)$, there exists a $P_{p}$-open set $U$ of $X$ containing $x$ such that $f(U) \subseteq V$. If $f$ is $P_{p}$-continuous at every point $x$ of $X$, then it is called $P_{p}$-continuous.

Definition 5. [19] A function $f: X \rightarrow Y$ is called quasi $\theta$-continuous at a point $x \in X$ if for each $\theta$-open set $V$ of $Y$ containing $f(x)$, there exists a $\theta$-open set $U$ of $X$ containing $x$ such that $f(U) \subseteq V$.

Corollary 2. [10] Every quasi $\theta$-continuous is $P_{p}$-continuous.

Definition 6. [20] A space $X$ is said to be semi-regular if for any open set $U$ of $X$ and each point $x \in U$, there exists a regular open set $V$ of $X$ such that $x \in V \subseteq U$.

\section{Almost $P_{p}$-Continuous Functions}

In this section, we introduce the notions of almost $P_{p}$-Continuous functions by using $P_{p}$-open sets. Some properties and characterizations are given. 
Definition 7. A function $f: X \rightarrow Y$ is called almost $P_{p}$-continuous at a point $x \in X$ if for each open set $V$ of $Y$ containing $f(x)$, there exists a $P_{p}$-open set $U$ of $X$ containing $x$ such that $f(U) \subseteq \operatorname{Int} C l(V)$. If $f$ is almost $P_{p}$-continuous at every point $x$ of $X$, then it is called almost $P_{p}$-continuous.

Lemma 3. The following results follows directly from their definitions:

(1) Every $P_{p}$-continuous function is almost $P_{p}$-continuous.

(2) Every almost $P_{p}$-continuous function is almost precontinuous.

Corollary 3. labelcsh Every quasi $\theta$-continuous function is almost $P_{p}$-continuous.

Proof. Follows from Corollary 2 and Lemma 3.

From Lemma 3, Corollary 2 and Corollary 5.4 in [10], the following diagram is obtained:

In the sequel, we shall show that none of the implications that concerning almost $P_{p^{-}}$- continuity in Diagram 1 is reversible.

Example 1. Consider $X=\{a, b, c, d\}$ with the two topologies $\tau=\{\phi,\{a\},\{a, b\}$,

$\{c, d\},\{a, c, d\}, X\}$ and $\sigma=\{\phi,\{b\},\{b, d\},\{b, c, d\}, X\}, P_{p} O(X)=\{\phi,\{c\},\{d\}$,

$\{a, b\},\{c, d\},\{a, b, c\},\{a, b, d\}, X\}$. Let $f:(X, \tau) \rightarrow(X, \sigma)$ be the identity function. Then $f$ is almost $P_{p}$-continuous, but it is not $P_{p}$-continuous, because $\{b\}$ is an open set in $(X, \sigma)$ containing $f(b)=b$, there exists no $P_{p}$-open $U$ in $(X, \tau)$ containing $b$ such that $b \in f(U) \subseteq\{b\}$.

Example 2. Consider $X=\{a, b, c\}$ with the topology $\tau=\sigma=\{\phi,\{a\},\{b\}$,

$\{a, b\}, X\}$. Let $f:(X, \tau) \rightarrow(X, \sigma)$ be the identity function. Then $f$ is almost precontinuous, but it is not almost $P_{p^{-}}$ continuous, because $\{a\}$ is an open set in $(X, \sigma)$ containing $f(a)=a$, there exists no $P_{p}$-open $U$ in $(X, \tau)$ containing $a$ such that $a \in f(U) \subseteq \operatorname{Int} C l(\{a\})$.

Theorem 1. For a function $f:(X, \tau) \rightarrow(Y, \sigma)$, the following statements are equivalent:

(1) $f$ is almost $P_{p}$-continuous.

(2) For each $x \in X$ and each open set $V$ of $Y$ containing $f(x)$, there exists a $P_{p}$-open set $U$ in $X$ containing $x$ such that $f(U) \subseteq s C l(V)$.

(3) For each $x \in X$ and each regular open set $V$ of $Y$ containing $f(x)$, there exists a $P_{p}$-open set $U$ in $X$ containing $x$ such that $f(U) \subseteq V$.

(4) For each $x \in X$ and each $\delta$-open set $V$ of $Y$ containing $f(x)$, there exists a $P_{p}$-open set $U$ in $X$ containing $x$ such that $f(U) \subseteq V$.

Proof. (1) $\Rightarrow$ (2). Let $x \in X$ and let $V$ be any open set of $Y$ containing $f(x)$. By (1), there exists a $P_{p}$-open set $U$ of $X$ containing $x$ such that $f(U) \subseteq \operatorname{Int} C l(V)$. Since $V$ is open, hence $V$ is preopen. Therefore, by Lemma $1(2), f(U) \subseteq s C l(V)$. $(2) \Rightarrow(3)$. Follow directly from definition 7 and Lemma 1(2).

(3) $\Rightarrow(4)$. Let $x \in X$ and let $V$ be any $\delta$-open set of $Y$ containing $f(x)$. Then for each $f(x) \in V$, there exists an open set $G$ containing $f(x)$ such that $G \subseteq \operatorname{Int} C l(G) \subseteq V$. Since $\operatorname{Int} C l(G)$ is a regular open set of $Y$ containing $f(x)$, by (3), there exists a $P_{p}$-open set $U$ in $X$ containing $x$ such that $f(U) \subseteq \operatorname{Int} C l(G) \subseteq V$. This completes the proof.

$(4) \Rightarrow(1)$. Let $x \in X$ and let $V$ be any open set of $Y$ containing $f(x)$. Then $\operatorname{Int} C l(V)$ is $\delta$-open of $Y$ containing $f(x)$. By

(4), there exists a $P_{p}$-open set $U$ in $X$ containing $x$ such that $f(U) \subseteq \operatorname{Int} C l(V)$. Therefore, $f$ is almost $P_{p}$-continuous.

Theorem 2. For a function $f: X \rightarrow Y$, the following statements are equivalent:

(1) $f$ is almost $P_{p}$-continuous.

(2) $f^{-1}(\operatorname{Int} C l(V))$ is a $P_{p}$-open set in $X$, for each open set $V$ in $Y$. 
(3) $f^{-1}(C l I n t(F))$ is a $P_{p}$-closed set in $X$, for each closed set $F$ in $Y$.

(4) $f^{-1}(F)$ is a $P_{p}$-closed set in $X$, for each regular closed set $F$ of $Y$.

(5) $f^{-1}(V)$ is a $P_{p^{-}}$open set in $X$, for each regular open set $V$ of $Y$.

Proof. (1) $\Rightarrow(2)$. Let $V$ be any open set in $Y$. We have to show that $f^{-1}(\operatorname{Int} C l(V))$ is $P_{p}$-open in $X$. Let $x \in f^{-1}(\operatorname{Int} C l(V))$. Then $f(x) \in \operatorname{IntCl}(V)$ and $\operatorname{IntCl}(V)$ is a regular open set in $Y$. Since $f$ is almost $P_{p}$-continuous, by Theorem 1 , there exists a $P_{p}$-open set $U$ of $X$ containing $x$ such that $f(U) \subseteq \operatorname{Int} C l(V)$. Which implies that $x \in U \subseteq f^{-1}(\operatorname{Int} C l V)$. Therefore, $f^{-1}(\operatorname{Int} C l(V))$ is $P_{p}$-open in $X$.

$(2) \Rightarrow(3)$. Let $F$ be any closed set of $Y$. Then $Y-F$ is an open set of $Y$. By (2), $f^{-1}(\operatorname{Int} C l(Y \backslash F))$ is $P_{p}$-open in $X$ and $f^{-1}(\operatorname{Int} C l(Y \backslash F))=f^{-1}(\operatorname{Int}(Y \backslash \operatorname{Int} F))=f^{-1}(Y \backslash C l \operatorname{Int} F)=X \backslash f^{-1}(C l \operatorname{Int} F)$ is $P_{p^{-}}$-open in $X$ and hence $f^{-1}(C l \operatorname{Int}(F))$ is $P_{p}$-closed in $X$.

$(3) \Rightarrow(4)$. Let $F$ be any regular closed set of $Y$. Then $F$ is a closed set of $Y$. By (3), $f^{-1}(C l \operatorname{Int}(F))$ is $P_{p}$-closed in $X$. Since $F$ is regular closed set, then $f^{-1}(\operatorname{ClInt}(F))=f^{-1}(F)$. Therefore, $f^{-1}(F)$ is $P_{p}$-closed set in $X$.

$(4) \Rightarrow(5)$. Let $V$ be any regular open set of $Y$. Then $Y \backslash V$ is regular closed of $Y$ and by (4), we have $f^{-1}(Y \backslash V)=$ $X \backslash f^{-1}(V)$ is $P_{p}$-closed in $X$ and hence $f^{-1}(V)$ is $P_{p}$-open in $X$.

$(5) \Rightarrow(1)$. Let $x \in X$ and let $V$ be any regular open set of $Y$ containing $f(x)$. Then $x \in f^{-1}(V)$. By (5), we have $f^{-1}(V)$ is $P_{p}$-open in $X$. Therefore, we obtain $f\left(f^{-1}(V)\right) \subseteq V$. Hence, by Theorem $1, f$ is almost $P_{p}$-continuous.

Theorem 3. For a function $f:(X, \tau) \rightarrow(Y, \sigma)$, the following statements are equivalent:

(1) $f$ is almost $P_{p}$-continuous.

(2) $f\left(P_{p} C l(A)\right) \subseteq C l_{\delta} f(A)$, for each subset $A$ of $X$.

(3) $P_{p} C l\left(f^{-1}(B)\right) \subseteq f^{-1}\left(C l_{\delta}(B)\right)$, for each subset $B$ of $Y$.

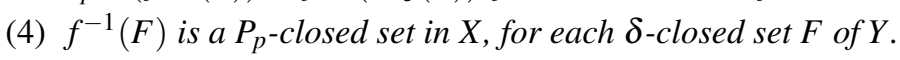

(5) $f^{-1}(V)$ is a $P_{p^{-o p e n}}$ set in $X$, for each $\delta$-open set $V$ of $Y$.

(6) $f^{-1}\left(\operatorname{Int}_{\delta}(B)\right) \subseteq P_{p} \operatorname{Int}\left(f^{-1}(B)\right)$, for each subset $B$ of $Y$.

(7) $\operatorname{Int}_{\delta}(f(A)) \subseteq f\left(P_{p} \operatorname{Int}(A)\right)$, for each subset $A$ of $X$.

Proof. (1) $\Rightarrow(2)$. Let $A$ be a subset of $X$. Since $C l_{\delta} f(A)$ is $\delta$-closed in $Y$. By (1) and Theorem $2, f^{-1}\left(C l_{\delta} f(A)\right)$ is $P_{p^{-}}$ closed set of $X$. Hence, $P_{p} C l A \subseteq f^{-1}\left(C l_{\delta} f(A)\right)$. Therefore, we obtain that $f\left(P_{p} C l A\right) \subseteq C l_{\delta} f(A)$.

$(2) \Rightarrow(3)$. Let $B$ be any subset of $Y$. Then $f^{-1}(B)$ is a subset of $X$. By (2), we have $f\left(P_{p} C l f^{-1}(B)\right) \subseteq C l_{\delta} f\left(f^{-1}(B)\right)=$ $C l_{\delta} B$. Hence, $P_{p} C l f^{-1}(B) \subseteq f^{-1}\left(C l_{\delta} B\right)$.

$(3) \Rightarrow(4)$. Let $F$ be any $\delta$-closed set of $Y$. By (3), we have $P_{p} C l f^{-1}(F) \subseteq f^{-1}\left(C l_{\delta} F\right)=f^{-1}(F)$ and hence $f^{-1}(F)$ is $P_{p}$-closed in $X$.

$(4) \Rightarrow(5)$. Let $V$ be any $\delta$-open set of $Y$. Then $Y \backslash V$ is $\delta$-closed of $Y$ and by (4), we have $f^{-1}(Y \backslash V)=X \backslash f^{-1}(V)$ is $P_{p}$-closed in $X$. Hence $f^{-1}(V)$ is $P_{p}$-open in $X$.

$(5) \Rightarrow(6)$. For each subset $B$ of $Y$. We have $\operatorname{Int}_{\delta} B \subseteq B$. Then $f^{-1}\left(\operatorname{Int}_{\delta} B\right) \subseteq f^{-1}(B)$. By (5), $f^{-1}\left(\operatorname{Int}_{\delta} B\right)$ is $P_{p}$-open in $X$. Then $f^{-1}\left(\operatorname{Int}_{\delta} B\right) \subseteq P_{p} \operatorname{Int} f^{-1}(B)$.

$(6) \Rightarrow(7)$. Let $A$ be any subset of $X$. Then $f(A)$ is a subset of $Y$. By (6), we have $f^{-1}\left(\operatorname{Int}_{\delta}(f(A)) \subseteq P_{p} \operatorname{Int}\left(f^{-1}(f(A)) \subseteq\right.\right.$ $P_{p} \operatorname{Int}(A)$. Therefore, $\operatorname{Int}_{\delta}(f(A)) \subseteq f\left(P_{p} \operatorname{Int}(A)\right)$.

$(7) \Rightarrow(1)$. Let $x \in X$ and let $V$ be any regular open set of $Y$ containing $f(x)$. Then $x \in f^{-1}(V)$ and $f^{-1}(V)$ is a subset of $X$. By (7), we have $\operatorname{Int}_{\delta}\left(f\left(f^{-1}(V)\right) \subseteq f\left(P_{p} \operatorname{Int}\left(f^{-1}(V)\right)\right.\right.$ implies that $\operatorname{Int}_{\delta}(V) \subseteq f\left(P_{p} \operatorname{Int}\left(f^{-1}(V)\right)\right.$. Since $V$ is regular open and hence it is $\delta$-open, then $V \subseteq f\left(P_{p} \operatorname{Int}\left(f^{-1}(V)\right)\right.$. This implies that $f^{-1}(V) \subseteq P_{p} \operatorname{Int}\left(f^{-1}(V)\right)$. Therefore, $f^{-1}(V)$ is a $P_{p}$-open set in $X$ which contains $x$ and clearly $f\left(f^{-1}(V)\right) \subseteq V$. Hence, by Theorem $1, f$ is almost $P_{p}$-continuous.

Theorem 4. For a function $f: X \rightarrow Y$, the following statements are equivalent:

(1) $f$ is almost $P_{p^{-} \text {-continuous. }}$

(2) $P_{p} C l f^{-1}(V) \subseteq f^{-1}(C l V)$, for each $\beta$-open set $V$ of $Y$. 
(3) $f^{-1}(\operatorname{Int}(F)) \subseteq P_{p} \operatorname{Int}\left(f^{-1}(F)\right)$, for each $\beta$-closed set $F$ of $Y$.

(4) $f^{-1}(\operatorname{Int}(F)) \subseteq P_{p} \operatorname{Int}\left(f^{-1}(F)\right)$, for each semi-closed set $F$ of $Y$.

(5) $P_{p} C l f^{-1}(V) \subseteq f^{-1}(C l V)$, for each semi-open set $V$ of $Y$.

Proof. (1) $\Rightarrow(2)$. Let $V$ be any $\beta$-open set of $Y$. By Lemma 1(3) that $C l(V)$ is regular closed in $Y$. Since $f$ is almost $P_{p}$-continuous, by Theorem $2, f^{-1}(C l V)$ is $P_{p}$-closed set in $X$. Therefore, we obtain $P_{p} C l f^{-1}(V) \subseteq f^{-1}(C l V)$.

(2) $\Rightarrow$ (3). Let $\mathrm{F}$ be any $\beta$-closed of $Y$. Then $Y \backslash F$ is $\beta$-open of $Y$ and by (2), we have $P_{p} C l f^{-1}(Y \backslash F) \subseteq f^{-1}(C l(Y \backslash F))$ and $P_{p} C l\left(X \backslash f^{-1}(F)\right) \subseteq f^{-1}(Y \backslash \operatorname{Int} F)$ and hence, $X \backslash P_{p} \operatorname{Int} f^{-1}(F) \subseteq X \backslash f^{-1}(\operatorname{Int} F)$. Therefore, $f^{-1}(\operatorname{Int} F) \subseteq P_{p} \operatorname{Int} f^{-1}(F)$.

(3) $\Rightarrow$ (4). Obvious since every semi-closed set is $\beta$-closed.

(4) $\Rightarrow(5)$. Let $V$ be any semi-open set of $Y$. Then $Y \backslash V$ is semi-closed in $Y$ and by (4), we have $f^{-1}(\operatorname{Int}(Y \backslash V)) \subseteq P_{p} \operatorname{Int} f^{-1}(Y \backslash V)$ and $f^{-1}(Y \backslash C l V) \subseteq P_{p} \operatorname{Int}\left(X \backslash f^{-1}(V)\right)$ and hence, $X \backslash f^{-1}(C l V) \subseteq X \backslash P_{p} C l f^{-1}(V)$. Therefore, $P_{p} C l f^{-1}(V) \subseteq f^{-1}(C l V)$.

$(5) \Rightarrow(1)$. Let $F$ be any regular closed set of $Y$. Then $F$ is a semi-open set of $Y$. By (5), we have $P_{p} C l f^{-1}(F) \subseteq f^{-1}(C l F)=f^{-1}(F)$. This shows that $f^{-1}(F)$ is a $P_{p}$-closed set in $X$. Therefore, by Theorem $2, f$ is almost $P_{p}$-continuous.

Theorem 5. For a function $f: X \rightarrow Y$, the following statements are equivalent:

(1) $f$ is almost $P_{p}$-continuous.

(2) $P_{p} C l f^{-1}(V) \subseteq f^{-1}(\alpha C l V)$, for each $\beta$-open set $V$ of $Y$.

(3) $P_{p} C l f^{-1}(V) \subseteq f^{-1}\left(C l_{\delta} V\right)$, for each $\beta$-open set $V$ of $Y$.

(4) $P_{p} C l f^{-1}(V) \subseteq f^{-1}\left(P_{p} C l V\right)$, for each semi-open set $V$ of $Y$.

(5) $P_{p} C l f^{-1}(V) \subseteq f^{-1}(p C l(V)$, for each semi-open set $V$ of $Y$.

Proof. (1) $\Rightarrow$ (2). Follows from Theorem 4 and Lemma 2(2).

(2) $\Rightarrow$ (3). Follows from the fact that $\alpha C l V \subseteq C l_{\delta} V$.

(3) $\Rightarrow(4)$ and $(4) \Rightarrow(5)$. Follows from Theorem 4 and Lemma 2(1).

$(5) \Rightarrow(1)$. Follows from Theorem 4 and Lemma 2(1).

The following result also can be concluded directly.

Corollary 4. For a function $f: X \rightarrow Y$, the following statements are equivalent:

(1) $f$ is almost $P_{p}$-continuous.

(2) $f^{-1}(\alpha \operatorname{Int} F) \subseteq P_{p}$ Int $f^{-1}(F)$, for each $\beta$-closed set $F$ of $Y$.

(3) $f^{-1}\left(\operatorname{Int}_{\delta} F\right) \subseteq P_{p} \operatorname{Intf}^{-1}(F)$, for each $\beta$-closed set $F$ of $Y$.

(4) $f^{-1}\left(P_{p}\right.$ Int $\left.F\right) \subseteq P_{p}$ Int $f^{-1}(F)$, for each semi-closed set $F$ of $Y$.

(5) $f^{-1}(p \operatorname{Int} F) \subseteq P_{p}$ Int $f^{-1}(F)$, for each semi-closed set $F$ of $Y$.

Theorem 6. A function $f: X \rightarrow Y$ is almost $P_{p}$-continuous if and only if $f^{-1}(V) \subseteq P_{p}$ Int $f^{-1}($ IntClV $)$ for each preopen set $V$ of $Y$.

Proof. Necessity. Let $V$ be any preopen set of $Y$. Then $V \subseteq \operatorname{Int} C l V$ and IntClV is a regular open set in $Y$. Since $f$ is almost $P_{p}$-continuous, by Theorem $2, f^{-1}(\operatorname{Int} C l V)$ is $P_{p}$-open in $X$ and hence we obtain that $f^{-1}(V) \subseteq f^{-1}(\operatorname{Int} C l V)=P_{p} \operatorname{Int} f^{-1}(\operatorname{Int} C l V)$.

Sufficiency. Let $V$ be any regular open set of $Y$. Then $V$ is a preopen set of $Y$. By hypothesis, we have $f^{-1}(V) \subseteq P_{p}$ Int $f^{-1}(\operatorname{Int} C l V)=P_{p} \operatorname{Int} f^{-1}(V)$. Therefore, $f^{-1}(V)$ is $P_{p}$-open in $X$ and hence by Theorem $2, f$ is almost $P_{p}$-continuous.

Corollary 5. The following statements are equivalent for a function $f: X \rightarrow Y$ : 
(1) $f$ is almost $P_{p}$-continuous.

(2) $f^{-1}(V) \subseteq P_{p}$ Int $^{-1}(s C l V)$ for each preopen set $V$ of $Y$.

(3) $P_{p} C l f^{-1}(C l I n t F) \subseteq f^{-1}(F)$ for each preclosed set $F$ of $Y$.

(4) $P_{p} C l f^{-1}(\operatorname{sint} F) \subseteq f^{-1}(F)$ for each preclosed set $F$ of $Y$.

Corollary 6. For a function $f: X \rightarrow Y$, the following statements are equivalent:

(1) $f$ is almost $P_{p}$-continuous.

(2) For each neighborhood $V$ of $f(x), x \in P_{p}$ Int $f^{-1}(s C l V)$.

(3) For each neighborhood $V$ of $f(x), x \in P_{p} \operatorname{Int}(\operatorname{IntClV})$.

Proof. Follows from Theorem 6 and Corollary 5.

Theorem 7. Let $f: X \rightarrow Y$ be an almost $P_{p}$-continuous function and let $V$ be any open subset of $Y$. If $x \in P_{p} C l f^{-1}(V) \backslash$ $f^{-1}(V)$, then $f(x) \in P_{p} C l V$.

Proof. Let $x \in X$ be such that $x \in P_{p} C l f^{-1}(V) \backslash f^{-1}(V)$ and suppose $f(x) \notin P_{p} C l V$. Then there exists a $P_{p}$-open set $H$ containing $f(x)$ such that $H \cap V=\phi$. Then $C l H \cap V=\phi$ implies Int $C l H \cap V=\phi$ and IntClH is a regular open set. Since $f$ is almost $P_{p}$-continuous, by Theorem 1 , there exists a $P_{p}$-open set $U$ in $X$ containing $x$ such that $f(U) \subseteq \operatorname{IntClH}$. Therefore, $f(U) \cap V=\phi$. However, since $x \in P_{p} C l f^{-1}(V), U \cap f^{-1}(V) \neq \phi$ for every $P_{p}$-open set $U$ in $X$ containing $x$, so that $f(U) \cap V \neq \phi$. We have a contradiction. It follows that $f(x) \in P_{p} C l V$.

Theorem 8. If $f: X \rightarrow Y$ is almost $P_{p}$-continuous and $g: Y \rightarrow Z$ is super continuous function, then the composition function $g \circ f: X \rightarrow Z$ is $P_{p}$-continuous.

Proof. Let $W$ be any open subset of $Z$. Since $g$ is super continuous, $g^{-1}(W)$ is $\delta$-open of $Y$. Since $f$ is almost $P_{p}$-continuous, by Theorem 3, $(g \circ f)^{-1}(W)=f^{-1}\left(g^{-1}(W)\right)$ is $P_{p}$-open in $X$. Therefore, by Definition $4, g \circ f$ is $P_{p}$-continuous.

Theorem 9. If $f: X \rightarrow Y$ is almost $P_{p}$-continuous and $g: Y \rightarrow Z$ is continuous and open, then the composition function $g \circ f: X \rightarrow Z$ is almost $P_{p}$-continuous.

Proof. Let $x \in X$ and $W$ be an open set of $Z$ containing $g(f(x))$. Since $g$ is continuous, $g^{-1}(W)$ is an open set of $Y$ containing $f(x)$. Since $f$ is almost $P_{p}$-continuous, there exists a $P_{p}$-open set $U$ of $X$ containing $x$ such that $f(U) \subseteq \operatorname{Int}\left(C l\left(g^{-1}(W)\right)\right)$. Also, since $g$ is continuous, then we obtain $(g \circ f)(U) \subseteq g\left(\operatorname{Int}\left(g^{-1}(C l(W))\right)\right)$. Since $g$ is open, we obtain $(g \circ f)(U) \subseteq$ $\operatorname{Int}(\mathrm{Cl}(\mathrm{W}))$. Therefore, $g \circ f$ is almost $P_{p}$-continuous.

Theorem 10. If $f: X \rightarrow Y$ is an almost $P_{p}$-continuous function and $Y$ is semi-regular, then $f$ is $P_{p}$-continuous.

Proof. Let $x \in X$ and let $V$ be any open set of $Y$ containing $f(x)$. By the semi-regularity of $Y$, there exists a regular open set $G$ of $Y$ such that $f(x) \in G \subseteq V$. Since $f$ is almost $P_{p}$-continuous, by Theorem 1, there exists a $P_{p}$-open set $U$ of $X$ containing $x$ such that $f(U) \subseteq G \subseteq V$. Therefore, $f$ is $P_{p}$-continuous.

Proposition 2. If $f: X \rightarrow Y$ is an almost $P_{p}$-continuous function and $g: Y \rightarrow Z$ a strongly $\theta$-continuous function, then $g \circ f: X \rightarrow Z$ is almost $P_{p}$-continuous.

Proof. Let $W$ be an open subset of $Z$. In view of strong $\theta$-continuity of $g, g^{-1}(W)$ is a $\theta$-open subset of $Y$. Again, since $f$ is almost $P_{p}$-continuous, $(g \circ f)^{-1}(W)=f^{-1}\left(g^{-1}(W)\right)$ is $P_{p}$-open in $X$. Hence, $g \circ f$ is almost $P_{p}$-continuous.

Theorem 11. Let $f: X \rightarrow Y$ be almost $P_{p}$-continuous. If $Y$ is a preopen subset of $Z$, then $f: X \rightarrow Z$ is almost $P_{p}$-continuous.

Proof. Let $V$ be any regular open set of $Z$. Since $Y$ is preopen, by Lemma 1(1), $V \cap Y$ is a regular open set in $Y$. Since $f: X \rightarrow Y$ is almost $P_{p}$-continuous, by Theorem $2, f^{-1}(V \cap Y)$ is a $P_{p}$-open set in $X$. But $f(x) \in Y$ for each $x \in X$. Thus $f^{-1}(V)=f^{-1}(V \cap Y)$ is a $P_{p}$-open set of $X$. Therefore, by Theorem $2, f: X \rightarrow Z$ is almost $P_{p}$-continuous. 
Corollary 7. Let $f: X \rightarrow Y$ be a function and let $X$ be a pre- $T_{1}$ space. Then $f$ is almost precontinuous if and only if $f$ is almost $P_{p}$-continuous.

Proof. Follows from Proposition 1(1).

Corollary 8. Let $f: X \rightarrow Y$ be a function and let $X$ be a pre- $R_{0}$ space. Then $f$ is almost precontinuous if and only if $f$ is almost $P_{p}$-continuous.

Proof. Follows from Corollary 1.

Corollary 9. Let $f: X \rightarrow Y$ be a function and let $X$ be a pre-regular space. If $f$ is almost continuous, then $f$ is almost $P_{p}$-continuous.

Proof. Follows from Proposition 1(2).

Corollary 10. Let $f: X \rightarrow Y$ be a function and let $X$ be a locally indiscrete space. Then $f$ is almost precontinuous if and only if $f$ is almost $P_{p}$-continuous.

Proof. Follows from Proposition 1(3).

Theorem 12. If a function $f: X \rightarrow Y$ is almost strongly $\theta$-continuous, then $f$ is almost $P_{p}$-continuous.

Proof. Let $V$ be any regular open set of $Y$. Since $f$ is almost strongly $\theta$-continuous, so $f^{-1}(V)$ is $\theta$-open and hence it is $P_{p}$-open. Therefore, by Theorem $2, f$ is almost $P_{p}$-continuous.

\section{Competing interests}

The authors declare that they have no competing interests.

\section{Authors' contributions}

All authors have contributed to all parts of the article. All authors read and approved the final manuscript.

\section{References}

[1] M. E. Abd El-Monsef, S. N. El-Deeb and R. A. Mahmoud, $\beta$-open sets and $\beta$-continuous mappings, Bull. Fac. Sci. Assuit. Univ., 12 (1) (1983), 1-18.

[2] A. S. Abdulla, On some applications of special subsets in topology, Ph. D. Thesis, Tanta Univ., 1986.

[3] D. Andrijevic, Semi-preopen sets, Math. Vesnik, 38 (1986), 24-36..

[4] M. Caldas, S. Jafari and T. Noiri, Characterizations of Pre- $R_{0}$ and Pre- $R_{1}$ Topological Spaces, Topology Proceedings, Vol. 25, Summer (2000), 17-30.

[5] J. Dontchev, Survey on preopen sets, The Proceedings of the Yatsushiro Topological Conference, (1998), 1-18.

[6] J. Dontchev, M. Ganster and T. Noiri. On p-closed spaces, Internat. J. Math. and Math. Sci., 24 (3) (2000), 203-212.

[7] S. Fomin, Extensions of topological spaces, Ann. of Math., 2 (44) (1943), 471-480.

[8] D. S. Jankovic, A note on mappings of extremally disconnected spaces, Acta Math. Hungar., 46 (1985), 83-92.

[9] A. Kar and P. Bhattacharyya, Some weak separation axioms, Bull. Cal. Math. Soc., 82 (1990), 415-422.

[10] A. B. Khalaf and Sh. M. Mershkhan, $P_{p}$-open sets and $P_{p}$-continuous Functions, Gen. Math. Notes, 20 (2014), 34-51.

[11] N. Levine, Semi-open sets and semi-continuity in topological spaces, Amer. Math. Monthly, 70 (1) (1963), 36-41.

[12] P. E. Long and L. Herrington, Strongly $\theta$-continuous functions, J. Korean Math. Soc., 18 (1) (1981), 21-28. 
[13] A. S. Mashhour, M.E. Abd El-Monsef and S.N. El-Deeb, On precontinuous and week precontinuous mappings, Proc. Math. Phys. Soc. Egypt, 53 (1982), 47-53.

[14] Sh. M. Mershkhan, Application of $P_{p}$-open sets in topological spaces, M. Sc. Thesis, Zakho University 2013.

[15] B. M. Munshi and D. S. Bassan, Super continuous functions, Indian J. Pure Appl. Math., 13 (1982), 229-236.

[16] A. A. Nasef and T. Noiri, Some weak forms of almost continuity, Acta Math. Hungar., 74 (1997), 211-219.

[17] O. Njastad, On some classes of nearly open sets, Pacific J. Math., 15 (3) (1965), 961-970.

[18] T. Noiri and S. M. Kang, On almost strongly $\theta$-continuous functions, Indian J. Pure Appl. Math., 15 (1984), 1-8.

[19] T. Noiri and V. Popa, Weak forms of faint continuity, Bull. Math. Soc. Sci. Math. Roumanie, 34 (82) (1990), 263-270.

[20] T. Noiri and V. Popa. On almost $\beta$-continuous functions, Acta Math. Hungar., 79 (4)(1998), 329-339.

[21] M. Pal and P. Bhattacharyya, Feeble and strong forms of pre-irresolute function, Bull. Malaysian Math. Soc. (Second Series), 19 (1996), 63-75.

[22] M. H. Stone, Applications of the theory of boolean rings to topology, Trans. Amer. Math. Soc., 41 (1937), 375-481.

[23] N. V. Velicko, H-closed topological spaces, Amer. Math. Soc. Transl., 78 (2) (1968), 103-118.

[24] R. H. Yunis. Some equivalent concepts defined by using $\alpha$-open sets in topological spaces, Zanco J. of Pure and Applied Sciences, 17 (1) (2005), 21-26. 